\title{
Quantum Field Theory for Orthofermions and Orthobosons
}

\author{
A. K. Mishra ${ }^{* \dagger}$ and G. Rajasekaran ${ }^{\dagger}$ \\ * Max-Planck Institute for Physics of Complex Systems, Nothnitzer Str. 38, D-01187 \\ Dresden, Germany, and \\ $\dagger$ Institute of Mathematical Sciences, CIT Campus, Madras - 600 113, India \\ e-mail: mishra@imsc.ernet.in; graj@imsc.ernet.in
}

\begin{abstract}
Orthofermi statistics is characterized by an exclusion principle which is more "exclusive" than Pauli's exclusion principle: an orbital state shall not contain more than one particle, no matter what the spin direction is. The wavefunction is antisymmetric in spatial indices alone with arbitrary symmetry in the spin indices. Orthobose statistics is corresponding Bose analog: the wavefunction is symmetric in spatial indices, with arbitrary symmetry in spin indices. We construct the quantum field theory of particles obeying these new kinds of quantum statistics. Non-relativistic as well as relativistic quantum field theories with interactions are considered.
\end{abstract}




\section{Introduction}

The constraint that an orbital state shall not contain more than one particle irrespective of their spin directions has led us to the formulation of a new family of quantum statistics, namely, orthostatistics [1]. These statistics are described through the algebra

$$
\begin{aligned}
c_{k \alpha} c_{p \beta}^{\dagger} \pm \delta_{\alpha \beta} \sum_{\gamma} c_{p \gamma}^{\dagger} c_{k \gamma} & =\delta_{k p} \delta_{\alpha \beta} \\
c_{k \alpha} c_{p \beta} \pm c_{p \alpha} c_{k \beta} & =0
\end{aligned}
$$

where $c_{k \alpha}$ and $c_{p \beta}^{\dagger}$ are the annihilation and creation operators of particles with momenta $k$ and $p$ and spins $\alpha$ and $\beta$ respectively. The positive sign corresponds to the orthofermi and negative sign is for the orthobose statistics.

Greenberg has earlier constructed infinite statistics wherein wavefunctions with arbitrary symmetries are allowed $[2,3]$. Infinite statistics also follows from Eq.(1) if the spatial indices $k$ and $p$ are suppressed [4]. On the other hand, if spin indices are suppressed, canonical commutation relations for boson and anticommutaors for fermion are obtained. Thus orthostatistics describe a generalized class of statistics wherein different indices exhibit different and uncorrelated symmetry properties.

One of the important property of orthofermions is that only one particle can be accommodated among the set of states $\{k \alpha\}$, irrespective of the range of $\alpha$. This contrast with the occupancy status of parafermions. In parafermi statistics of order $n$, utmost $n$ parafermions can occupy the same state [5].

Until now, it has been possible to construct a local relativistic quantum field theory (LRQFT) only for parafermions and parabosons, of which fermion and boson are specific examples [6]. No such formulation is possible for infinite statistics [2,3]. Since the orthofermions (orthobosons) have properties common with fermions (bosons) as well as with the particles obeying infinite statistics, we are motivated to examine whether a LRQFT for orthoparticles can be constructed? We show here that if the second index $\alpha$ in $c_{k \alpha}$ is reinterpreted as a new degree of freedom which can be excited at some higher energy scale yet to be probed experimentally, it is possible to construct LRQFT for orthofermions [7]. However, the relativistic quantum field theory for the orhobosons remains nonlocal.

The nonrelativistic quantum field theory for the both kinds of particles can be constructed even when $\alpha$ denotes the usual kinematic spin variable. This is considered in Sec.2. 
Formulations of Dirac field as an orthofermi field and Klein-Gordon field as an orthobose field, provided $\alpha$ characterizes the new degree of freedom, are given in sections 3 and 4 , respectively. Interactions are considered in Sec.5, followed by a section on summary and conclusions.

\section{Nonrelativistic Quantum Field theory}

The orthoparticles obey quantization rules different from the canonical commutators and anticommutators. The Poisson brackets involving canonical coordinate $\psi$ and canonical momentum $\pi$, that is, $[\psi, \pi]_{P B}$ and $[\psi, \psi]_{P B}$ are now replaced by the new quantum brackets corresponding to Eqs.(1) and (2), or equivalently

$$
\begin{gathered}
\psi_{a}(\mathbf{x}, 0) \psi_{b}^{\dagger}(\mathbf{y}, 0) \pm \delta_{a b} \sum_{c} \psi_{c}^{\dagger}(\mathbf{y}, 0) \psi_{c}(\mathbf{x}, 0)=\delta_{a b} \delta^{3}(\mathbf{x}-\mathbf{y}) \\
\psi_{a}(\mathbf{x}, 0) \psi_{b}(\mathbf{y}, 0) \pm \psi_{a}(\mathbf{y}, 0) \psi_{b}(\mathbf{x}, 0)=0
\end{gathered}
$$

In spite of different quantization procedure, the corresponding nonrelativistic field theory can be developed in a consistent manner. Consider the nonrelativistic field $\psi_{a}(\mathbf{x}, t)$ satisfying the Schrodinger equation

$$
\left(i \frac{\partial}{\partial t}+\frac{1}{2 m} \nabla^{2}\right) \psi_{a}(\mathbf{x}, t)=0
$$

The fourier expansion for $\psi_{a}$ is

$$
\psi_{a}(\mathbf{x}, t)=\sum_{\mathbf{k}, \alpha} c_{\mathbf{k} \alpha} \xi_{a}^{(\alpha)} e^{\mathbf{i k} \cdot \mathbf{x}-i \omega_{k} t},
$$

where $\xi_{a}^{(\alpha)}$ is the spin wavefunction for the spin-component $\alpha$, and $\omega_{k}=k^{2} /(2 m)$ is the nonrelativistic energy.

The Hamiltonian of the system is

$$
H=\sum_{\mathbf{k}, \alpha} \omega_{k} c_{\mathbf{k} \alpha}^{\dagger} c_{\mathbf{k} \alpha}
$$

From Eqs.(1) and (2) (with either sign), the following commutation relation can be derived

$$
\left[c_{\mathbf{k} \alpha}, \sum_{\gamma} c_{\mathbf{p} \gamma}^{\dagger} c_{\mathbf{p} \gamma}\right]=\delta_{\mathbf{k p}} c_{\mathbf{k} \alpha}
$$


Now it can be shown that the Schrodinger Eq.(5) follows from the Heisenberg equation of motion for $\psi_{a}$ and the relation (8).

The Schrodinger equation and the Hamiltonian can also be obtained from the canonical formalism. In fact the Schrodinger Eq.(5) is the Euler-Lagrange equation of motion for the usual Lagrange density

$$
\mathcal{L}=i \sum_{a} \psi_{a}^{*} \frac{\partial \psi_{a}}{\partial t}-\frac{1}{2 m} \sum_{a} \nabla \psi_{a}^{*} \cdot \nabla \psi_{a}
$$

The Legendre transformation gives the corresponding total Hamiltonian as

$$
H=\int d^{3} x \mathcal{H}=\frac{1}{2 m} \int d^{3} x \sum_{a} \psi_{a}^{*} \nabla^{2} \psi_{a}
$$

Substitution of Eq.(6) into Eq.(10) leads to the Hamiltonian given by Eq.(7).

Thus the analysis given in this section shows that our quantization is consistent, and a nonrelativistic quantum field theory based on a canonical Lagrangian formalism can be constructed for the orthoparticles.

\section{Relativistic Quantum field theory for Orthofermions}

We consider the Dirac Hamiltonin

$$
H=\int \psi^{\dagger}(\boldsymbol{\alpha} \cdot \mathbf{p}+\beta m) \psi d^{3} x
$$

and the usual four component Dirac field $\psi_{a}(\mathbf{x}, t)(a=1,2,3,4)$. Using the expansion

$$
\psi_{a}(\mathbf{x}, t)=\sum_{\mathbf{k}} \sum_{\alpha=1}^{4}\left(\frac{m}{E_{k}}\right)^{1 / 2} c_{\mathbf{k} \alpha} u_{a}^{(\alpha)}(\mathbf{k}) e^{i \mathbf{k} \cdot \mathbf{x}-i E_{k}^{(\alpha)} t}
$$

and the relation

$$
(\boldsymbol{\alpha} \cdot \mathbf{k}+\beta m) u^{(\alpha)}(\mathbf{k})=E_{k}^{(\alpha)} u^{(\alpha)}(\mathbf{k})
$$

in Eq.(11), the Hamiltonian can be written as

$$
H=\sum_{k} E_{k}\left[\sum_{\alpha=1,2} c_{\mathbf{k} \alpha}^{\dagger} c_{\mathbf{k} \alpha}-\sum_{\alpha=3,4} c_{\mathbf{k} \alpha}^{\dagger} c_{\mathbf{k} \alpha}\right]
$$


$u_{a}^{(\alpha)}(\mathbf{k})$ in expansion (12) are Dirac spinors, $\alpha=1$ and 2 being the positive energy spinors $\left(E_{k}^{(\alpha)} \equiv E_{K}=+\left(m^{2}+k^{2}\right)^{2}\right)$, and $\alpha=3$ and 4 being the negative energy spinors $\left(E_{k}^{(\alpha)} \equiv\right.$ $\left.-E_{k}\right) . \boldsymbol{\alpha}, \beta$ are the Dirac matrices and $\mathbf{p}=-\mathrm{i} \nabla$.

If $c_{\mathbf{k} \alpha}$ and $c_{\mathbf{k} \alpha}^{\dagger}$ satisfy the orthofermionic algebra given in Eqs.(1) and (2), and the second index $\alpha$ for an orthfermion is identified as the Dirac index $\alpha$ going from 1 to 4 , then neither $\sum_{\alpha=1,2} c_{\mathbf{k} \alpha}^{\dagger} c_{\mathbf{k} \alpha}$ nor $\sum_{\alpha=3,4} c_{\mathbf{k} \alpha}^{\dagger} c_{\mathbf{k} \alpha}$ occurring in the Hamiltonian (Eq.(14)) are number operators. It can be verified from Eq.(8) that only the sum $\sum_{\alpha=1-4} c_{\mathbf{k} \alpha}^{\dagger} c_{\mathbf{k} \alpha}$ is a number operator for particles of momenta k. Therefore as such, the Hamiltonian in Eq.(14) can not be reexpressed as the sum of energies of particles, and our quantization procedure seems to fail. Note that if $c^{\dagger}$ and $c$ in Eq.(14) denote creation and annihilation operators for the usual fermion, the Hamiltonian can be reexpressed as the sum of energies of particles and antiparticles by introducing the Dirac vacuum state.

The above problem can be circumvented by introducing a new degree of freedom indexed by $A, B, C, D \ldots$ The orthofermi algebra with the new indices is written as

$$
\begin{aligned}
c_{\mathbf{k} \alpha A} c_{\mathbf{p} \beta B}^{\dagger}+\delta_{A B} \sum_{D} c_{\mathbf{p} \beta D}^{\dagger} c_{\mathbf{k} \alpha D} & =\delta_{\mathbf{k p}} \delta_{\alpha \beta} \delta_{A B} \\
c_{\mathbf{k} \alpha A} c_{\mathbf{p} \beta B}+c_{\mathbf{p} \beta A} c_{\mathbf{k} \alpha B} & =0
\end{aligned}
$$

The Eq.(14) for the Hamiltonian now gets replaced as

$$
H=\sum_{k} E_{k}\left[\sum_{\alpha=1,2} \sum_{A} c_{\mathbf{k} \alpha A}^{\dagger} c_{\mathbf{k} \alpha A}-\sum_{\alpha=3,4} \sum_{A} c_{\mathbf{k} \alpha A}^{\dagger} c_{\mathbf{k} \alpha A}\right]
$$

It can be verified that

$$
n_{\mathbf{k} \alpha}=\sum_{A} c_{\mathbf{k} \alpha A}^{\dagger} c_{\mathbf{k} \alpha A}
$$

is the number operator for particles of momentum $\mathbf{k}$ and Dirac index $\alpha$. Note that in orthostatistics, number operators $n_{\mathbf{k} \alpha A}$ with all the indices specified, do not exist [8]. The Dirac vacuum is introduced as the filled negative energy sea. The vacuum state is infinitely degenerate since orthofermions of negative (as well as positive) energy can have arbitrary index $A$. One way of lifting this degeneracy is to choose the vacuum state as the normalized sum of all the states with the new index taking the full range of values.

The creation and annihilation operators for antiparticles are defined as

$$
d_{\mathbf{k} \alpha A}=c_{\mathbf{k} \alpha+2, A}^{\dagger} ; d_{\mathbf{k} \alpha A}^{\dagger}=c_{\mathbf{k} \alpha+2, A} ; \alpha=1,2
$$


The number operator for antiparticles is

$$
\bar{n}_{\mathbf{k} \alpha}=1-n_{\mathbf{k} \alpha+2}=1-\sum_{A} c_{\mathbf{k} \alpha+2, A}^{\dagger} c_{\mathbf{k} \alpha+2, A} \quad \text { for } \alpha=1,2
$$

which can be rewritten as $\bar{n}_{\mathbf{k} \alpha}=d_{\mathbf{k} \alpha A}^{\dagger} d_{\mathbf{k} \alpha A}$, for any $A$. The $A$-independence of the product $d_{\mathbf{k} \alpha A}^{\dagger} d_{\mathbf{k} \alpha A}$ follows from the Eqs.(15) and (19). Using Eqs.(18) and (20), the Eq.(17) can be rewritten as

$$
H=\sum_{k} E_{k} \sum_{\alpha=1,2}\left(n_{k \alpha}+\bar{n}_{k \alpha}\right)-\sum_{k} E_{k} \sum_{\alpha=1,2} 1
$$

Note that no summation over $A$ appears in the last term describing the vacuum energy. In orthofermi statistics, for each $\mathbf{k}$ and $\alpha$, there is only one particle irrespective of the value of $A$. Subtracting the vacuum energy, we finally get the modified Hamiltonian

$$
\widetilde{H}=H-<0|H| 0>=\sum_{k} E_{k} \sum_{\alpha=1,2}\left(n_{\mathbf{k} \alpha}+\bar{n}_{\mathbf{k} \alpha}\right)
$$

which is positive definite and is expressed as the sum of energies of particles and antiparticles. That the Heisenberg equation of motion for $\psi_{a A}(\mathbf{x}, t)$ is consistent with the Dirac equation

$$
i \frac{\partial \psi_{a A}}{\partial t}=(\boldsymbol{\alpha} \cdot \mathbf{p}+\beta m) \psi_{a A}
$$

and the algebra of $c$ and $c^{\dagger}$ (Eq.(15), can be easily verified. The Hamiltonian (Eq.(17)) can also be derived from the Lagrangian density

$$
\mathcal{L}=\sum_{a A} i \psi_{a A}^{\dagger} \frac{\partial \psi_{a A}}{\partial t}-\sum_{a A} \psi_{a A}^{\dagger}(-i \boldsymbol{\alpha} \cdot \nabla+\beta m) \psi_{a A}
$$

Thus the consistency of quantization rule as given by Eqs. $(15,16)$, or equivalently by the relations

$$
\begin{gathered}
\psi_{a A}(\mathbf{x}, 0) \psi_{b B}^{\dagger}(\mathbf{y}, 0)+\delta_{A B} \sum_{D} \psi_{b D}^{\dagger}(\mathbf{y}, 0) \psi_{a D}(\mathbf{x}, 0)=\delta_{A B} \delta_{a b} \delta^{3}(\mathbf{x}-\mathbf{y}) \\
\psi_{a A}(\mathbf{x}, 0) \psi_{b B}(\mathbf{y}, 0)+\psi_{b A}(\mathbf{y}, 0) \psi_{a B}(\mathbf{x}, 0)=0
\end{gathered}
$$

is established.

In spite of the modified structure of the basic commutation relations in Eqs.(25) and (26), bilinear observables such as the current density $j_{\mu}(\mathbf{x}, t)$ at two different points commute for space like separation, thus satisfying the microcausality condition. We define the current 
density four-vector $j_{\mu}=\sum_{A} \psi_{A}^{\dagger} \gamma_{0} \gamma_{\mu} \psi_{A}$ where $\gamma_{0}=\beta$ and $\boldsymbol{\gamma}=\gamma_{0} \boldsymbol{\alpha}$. It is straightforward to show that

$$
\left[j_{\mu}(\mathbf{x}, 0), j_{\nu}(\mathbf{y}, 0)\right]=0 \text { for } x \neq y
$$

Because of the relativistic invariance, commutativity of $j_{\mu}(\mathbf{x}, t)$ and $j_{\nu}\left(\mathbf{y}, t^{\prime}\right)$ for arbitrary space like separations follows from the above relation.

\section{Scalar Field as an orthobose Field}

The Eqs.(15) and (16) with the positive signs being replaced by negative signs define the algebra for orthobosons. We consider a real (Hermitian) scalar field

$$
\phi_{A}(\mathbf{x}, t)=\phi_{A}^{\dagger}(\mathbf{x}, t)=\sum_{k} \frac{1}{\left(2 \omega_{k}\right)^{1 / 2}}\left(c_{\mathbf{k} A} e^{i \mathbf{k} \cdot \mathbf{x}-i \omega_{k} t}+c_{\mathbf{k} A}^{\dagger} e^{\left.-i \mathbf{k} \cdot \mathbf{x}+i \omega_{k} t\right)}\right.
$$

$\omega_{k}=+\left(k+m^{2}\right)^{1 / 2}$. The Hamiltonian is taken as

$$
H=\frac{1}{2} N \int \sum_{A}\left(\nabla \phi_{A} \cdot \nabla \phi_{A}+m^{2} \phi_{A} \phi_{A}+\dot{\phi}_{A} \dot{\phi}_{A}\right) d^{3} x=\sum_{\mathbf{k}} \omega_{k} n_{\mathbf{k}}
$$

where $N$ denotes the normal ordering operator. The number operator for definite momentum $\mathbf{k}$ is $n_{\mathbf{k} A}=\sum_{A} c_{\mathbf{k} A}^{\dagger} c_{\mathbf{k} A}$. If the Hamiltonian had been defined without normal ordering, $\omega_{k}$ in Eq.(29) would have been scaled by a term $(1+n) / 2$, where $n$ is the range of the $A$. The operator $N$ removes this unacceptable scaling term along with the zero point energy.

The consistency of the quantization can be established by showing that Klein-Gordon equation for $\phi$ follows from the Heisenberg equation of motion and orthobose algebra. The Hamiltonian density $\mathcal{H}(x)$ in Eq.(29) can also be derived by starting with the Lagrangian density

$$
\mathcal{L}=\sum_{A} \frac{1}{2}\left\{\frac{\partial \phi_{A}}{\partial t} \frac{\partial \phi_{A}}{\partial t}-\nabla \phi_{A} \cdot \nabla \phi_{A}-m^{2} \phi_{A} \phi_{A}\right\}
$$

and employing the Legendre transformation.

However, if we take the Hamiltonian density as a local operator, it can be shown that $\mathcal{H}(\mathbf{x}, 0)$ and $\mathcal{H}(\mathbf{y}, 0)$ do not commute for $\mathbf{x} \neq \mathbf{y}$. The commutation relations among $\phi^{S}$ needed for this purpose can be derived using the Eq.(28) and orthobose algebra. Thus the relativistic field theory for orthobose does not satisfy the microcausality. 


\section{Interactions}

The interactions can be introduced in the relativistic theory in usual way. A brief comment about the nature of new degree of freedom, which has been left unspecified so far, will help us in formulating appropriate interactions. We may consider that the new degree of freedom is also described by a compact Lie group symmetry such as $S U(n)$ or $S O(n)$ etc., so that the field $\psi_{A}$ and $\phi_{A}$ form representations of Lie algebra with index $A$ labelling the components of multiplet as in the usual quantum field theory. Of course, the physical consequences in the present case will be different.

The interaction terms can be now constructed which are invariant under the Lie group transformations. For example, if $\psi_{A}$ is an orthofermi field which is a multiplet under $S U(n)$, and $\phi$ is an ordinary Bose field which is singlet under $S U(n)$, an interaction term is given as $\sum_{A} \psi_{A}^{\dagger} \beta \psi_{A} \phi$.

\section{Summary and conclusions}

In contrast to infinite statistics, we have been able to construct local relativistic quantum field theory for orthofermions. The two factors responsible for this are: (i) the number operators are bilinear in $c^{\dagger}$ and $c$, and (ii) in orthofield theory, quadratic relation in $c$ exists.

However, our success has been achieved by introducing a new degree of freedom. In fact we have circumvented the problem faced by infinite statistics by allowing the conventional degrees of freedom to be associated with the fermionic (antisymmetry) or bosonic (symmetric) behaviour in the wave function and assigning the new property of infinite statistics (arbitrary symmetry) to the new degree of freedom.

It has been pointed out that the infinite statistics can not be based on a canonical Lagrangian formalism [9]. In contrast, orthofield theory is based on a canonical Lagrangian formalism for the Schrodinger and Dirac fields.

It is most remarkable that microcausality is satisfied for the orthofermi field. Thus, we have shown that even with a modified quantization procedure, it is possible to obtain a consistent quantum field theory, and thus the framework of quantum field theory has been enlarged. 


\section{References}

[1] Mishra A. K., and Rajasekaran G., Pramana - J. Phys. 36, 537-555 (1991); 37, 455 (E) (1991).

[2] Greenberg O. W., Phys. Rev. Lett. 64, 705-708 (1990).

[3] Greenberg O. W., Phys. Rev. D43, 4111-4120 (1991).

[4] Mishra A. K., and Rajasekaran G., Phys. Lett. A 188, 210-214 (1994).

[5] Green H. S., Phys. Rev. 90, 270-273 (1953).

[6] Fredenhagen K., Commun. Math. Phys. 79, 141-151 (1981).

[7] Mishra A. K., and Rajasekaran G., Mod. Phys. Lett. A 7, 3425-3437 (1992); 11, 1031 (E) (1996).

[8] Mishra A. K., and Rajasekaran G., Pramana - J. Phys. 45, 91-139 (1995); hepth/9605204.

[9] Chaturvedi S., Kapoor A. K., Sandhya R., Srinivasan V., and Simon R., Phys. Rev. A43, 4555-4557 (1991). 